\title{
VALENCE BAND QUANTIZATION IN A SPHERICAL QUANTUM DOT
}

\author{
R. Buczko \\ Institute of Physics, Polish Academy of Sciences \\ Al. Lotników 32/46, 02-668 Warszawa, Poland
}

\begin{abstract}
An analytical solution of the effective mass equation for a spherical valence band in cubic semiconductors is presented. The full degeneracy of the valence band, i.e. the light-hole, heavy-hole and spin-orbit split-off subbands, were taken into account. The analytical wave functions of the valence band were used for its quantization in a completely isolated (infinite potential barrier approximation) quantum dot. The dependence of discrete valence levels on the dot diameter is presented for the $\mathrm{CuCl}$ case.
\end{abstract}

PACS numbers: $73.20 . D x$

\section{Introduction}

In the last years the fabrication of heterostructures has been developed from the two-dimensional to one- or even quasi-zero-dimensional (dot semiconductors) microcrystallites. For the description of the electronic bands inside a dot the assumption of the single parabolic valence band is sufficient in many cases $[1,2]$. In the zinc-blende semiconductors, however, the mixing of the light and heavy hole states plays an important role [3-6]. In the cases when the spin-orbit (SO) splitting is small or the split subband lies above the $\Gamma_{8}$ one (as for CLCl case), there is a necessity to take into account also $\Gamma_{7}(\mathrm{SO})$ band. The last was done only for the one symmetry of the quantum state $[3,6]$. The main concern of this work is to present a simple, nearly analytical model of the quantization of the fully degenerate valence band. The model is an extension of the one proposed by Ekimov et al. [3], and it concerns the three bands and arbitrary symmetry of the confined state. For simplicity we assume that the hole is completely confined in the well by the infinite potential barrier and the radius of the dot is not too small, therefore the effective mass approximation can be used.

\section{Spherically symmetric eigenstates of the valence band in the bulk}

Let us assume the spherical symmetry of the band and look for the Schrödinger equation solution of the same symmetry. The following effective units: the effective Rydberg constant $R^{*}=e^{4} m_{0} /\left(2 \hbar^{2} \varepsilon_{0}^{2} \gamma_{1}\right)$ as the unit of energy and effective 
Bohr radius $a^{*}=\hbar^{2} \varepsilon_{0} \gamma_{1} /\left(e^{2} m_{0}\right)$ as the unit of length will be used in the following ( $\gamma_{1}$ is the Luttinger mass parameter). For the wave vector $k$ in the $z$ direction, the Luttinger kinetic energy matrix, written in the basis of the Bloch functions $\left(u_{3 / 2}^{3 / 2}, u_{1 / 2}^{3 / 2}, u_{-1 / 2}^{3 / 2}, u_{-3 / 2}^{3 / 2}\right)$ for the $\Gamma_{8}$ subband and $\left(u_{1 / 2}^{1 / 2}, u_{-1 / 2}^{1 / 2}\right)$ for SO subband, has the form

$$
\left(\begin{array}{cccc|cc}
-\alpha k^{2} & 0 & 0 & 0 & 0 & 0 \\
0 & -\beta k^{2} & 0 & 0 & -\mathrm{i} \sqrt{2} \mu k^{2} & 0 \\
0 & 0 & -\beta k^{2} & 0 & 0 & -\mathrm{i} \sqrt{2} \mu k^{2} \\
0 & 0 & 0 & -\alpha k^{2} & 0 & 0 \\
\hline 0 & \mathrm{i} \sqrt{2} \mu k^{2} & 0 & 0 & -k^{2}-\Delta & 0 \\
0 & 0 & \mathrm{i} \sqrt{2} \mu k^{2} & 0 & 0 & -k^{2}-\Delta
\end{array}\right)
$$

where $\mu=\left(4 \gamma_{2}+6 \gamma_{3}\right) / 5 \gamma_{1}, \alpha=1-\mu, \beta=1+\mu, \Delta$ is the energy of SO splitting, $\gamma_{1}, \gamma_{2}, \gamma_{3}$ are the Luttinger parameters.

All the eigenvalues of the above Hamiltonian are twofold degenerate: the heavy hole band energy $E_{\mathrm{hh}}(k)=-\alpha k^{2}$, for the eigenstates $\varphi_{\mathrm{hh}}=u_{ \pm 3 / 2}^{3 / 2} \mathrm{e}^{\mathrm{i} k z}$, and $E_{ \pm}(k)=-(1+\mu / 2) k^{2}-\Delta / 2 \pm \Delta \sqrt{1-2 \mu k^{2} / \Delta+9 k^{4} / \Delta^{2}} / 2$, for the eigenstates $\varphi_{ \pm}=\left(a_{ \pm} u_{1 / 2}^{3 / 2}+b_{ \pm} u_{1 / 2}^{1 / 2}\right) \mathrm{e}^{\mathrm{i} k z}$. Here the upper sign corresponds to the light-hole band and the lower to the SO band $\left(E_{\mathrm{lh}}(k)=E_{+}(k)\right.$ and $\left.E_{\mathrm{so}}(k)=E_{-}(k)\right)$. Two conditions must be satisfied by the coefficients $a$ and $b: a_{ \pm}=-\mathrm{i} \sqrt{2} \mu k^{2} b_{ \pm} /\left(\beta k^{2}+E_{ \pm}(k)\right)$ and $a_{ \pm}^{2}+b_{ \pm}^{2}=1$. Both $\pm(\mathrm{lh}, \mathrm{so})$ states have their Kramers conjugate partners. The non-parabolicity of the $E_{ \pm}(k)$ reflects the well-known effect of the mixing of the light-hole and SO bands.

Since the energies of the bands do not depend on the direction of the $k$ vector, it is possible to obtain the eigenstates of the plain wave character for any direction of $k$ by an appropriate rotation of the above solutions. By examining the transformation properties of the wave functions under rotations we can find their partial wave $(J, M, k, \sigma)$ representation. The parameters $J$ and $M$ are the total angular momentum and its projection on the $z$ direction and $\sigma$ is the parity of the function under inversion. The new spherical solutions are

$$
\Psi_{J M k \sigma}^{\mathrm{hh}}(r)=4 \sqrt{2} \sum_{l(\sigma)}(-1)^{\eta(l)} C_{J, \frac{3}{2}, \frac{3}{2},-\frac{3}{2}}^{l, 0} Y_{J, M}^{l, \frac{3}{2}}(\vartheta, \varphi) j_{l}(k r)
$$

for the heavy-hole band and $\Psi_{J M k \sigma}^{\mathrm{h}}=\Psi_{J M k \sigma}^{-}, \Psi_{J M k \sigma}^{\mathrm{so}}=\Psi_{J M k \sigma}^{+}$for the light-hole and SO bands, where

$$
\begin{aligned}
& \Psi_{J M k \sigma}^{ \pm}(r)=4 \sqrt{2} \sum_{l(\sigma)}(-1)^{\eta(l)} j_{l}(k r) \\
& \times\left[a_{ \pm}(k) C_{J, \frac{1}{2}, \frac{3}{2},-\frac{1}{2}}^{l, 0} Y_{J, M}^{l, \frac{3}{2}}(\vartheta, \varphi)-b_{ \pm}(k) C_{J, \frac{1}{2}, \frac{1}{2},-\frac{1}{2}}^{l, 0} Y_{J, M}^{l, \frac{1}{2}}(\vartheta, \varphi)\right] .
\end{aligned}
$$

$j_{l}(x)$ is the spherical Bessel function, $C_{j, M, j, j_{z}}^{l, m}$ are Clebsch-Gordan coefficients and $Y_{J, M}^{l, j}=\left(Y^{l} \otimes u^{j}\right)_{M}^{J}, Y^{l}$ denotes the spherical harmonic. The summation runs over even values of $l$ for an even state and over odd values for an odd one. For any value of $J \geq 1 / 2$ therc exist only two values of $l$ for which the Clebsch-Gordan coefficients are not equal to zero, for both even and odd states. For $J=1 / 2$ : in 
the case of the SO subband $l=0$ for even states and $l=1$ for the odd ones, whereas for the light hole subband $l=2$ or 1 , respectively. There exist no heavy hole solutions with the total momentum $J=1 / 2$. The exponent $\eta$ depends on $l$ and is equal to $l / 2$ for an even state and to $(l-1) / 2$ for an odd one. The obtained analytical solutions can be easily applied to any problem of the spherical symmetry. In particular, they can be used for the quantization of the energy levels inside a dot by standard methods.

\section{The quantization of the band inside the dot}

The confinement of the hole in the quantum dot is modelled by the potential of spherical symmetry, flat and equal to zero inside the sphere of the radius $R$ and infinite $(-\infty$ in the electron scale of energy) outside the dot. The translational symmetry is broken and the states with different $k$ numbers can mix together, nevertheless $J, M$ and $\sigma$ are still good quantum numbers. The eigenstates are described by the linear combinations of the bulk wave functions corresponding to the same bulk energy

and

$$
\Psi_{J M \sigma}^{E_{n}}=A \Psi_{J M k_{\mathrm{hh}} \sigma}^{\mathrm{hh}}+B \Psi_{J M k_{1 \mathrm{~h}} \sigma}^{\mathrm{hh}}+C \Psi_{J M k_{\mathrm{so}} \sigma}^{\mathrm{so}}
$$

$$
E_{n}=E_{\mathrm{hh}}\left(k_{\mathrm{hh}}\right)=E_{\mathrm{lh}}\left(k_{\mathrm{lh}}\right)=E_{\mathrm{so}}\left(k_{\mathrm{so}}\right) \text {. }
$$

One should be careful when $E$ lies between SO and $\Gamma_{8}$ subbands - it is necessary to use imaginary $k$ value for the lower band in the dispersion relation. The energy values do not constitute a band any more, only discrete levels are allowed in the totally limited space. The levels are determined by the boundary condition for the wave function (i.e. $\Psi_{J M \sigma}^{E_{n}}(R)=0$ ). In general, $\Psi_{J M \sigma}^{E_{n}}$ consists of three independent tensors $Y_{J, M}^{l, j}$, therefore the boundary condition is equivalent to three linear equations for the $A, B$, and $C$ coefficients. In the particular case $J=1 / 2$ we have only two equations, for $B$ and $C$ coefficients. The roots of the appropriate energy dependent determinant, which constitute the quantum levels of the dot, can be obtained by numerical calculations on a PC.

As an example the results of quantization of the band in the sophisticated material with $\Delta=5 R^{*}$ is presented in Fig. 1 . The dependence on $\mu$ of the energy of the first ten $J=3 / 2$ even states is presented in comparison with the results obtained without the mixing between $\mathrm{SO}$ and $\Gamma_{8}$ wave functions, but with the non-parabolicity of the SO and lh subbands. It can be observed that, besides the differences in energy values, the main effect of the mixing is the level anticrossing. The next example is the quantization of the valence band in the $\mathrm{CuCl}$ dot. The band parameters for this material are $R^{*}=257 \mathrm{meV}, a^{*}=2.8 \AA, \Delta=-0.25 R^{*}$, and $\mu=0.26$. The SO subband lies above the hh and lh subbands and one can expect that the admixture of the $\Gamma_{8}$ wave functions to the quantized upper SO levels is important. The results obtained for $J=1 / 2$ even states do not confirm this expectation. The state energies as functions of the $R$ diameter exhibit only a small anticrossing effect. However, in the case of $J=3 / 2$ states, in the energy region between the bands, a strong non-monotonical behaviour of the state energies can 

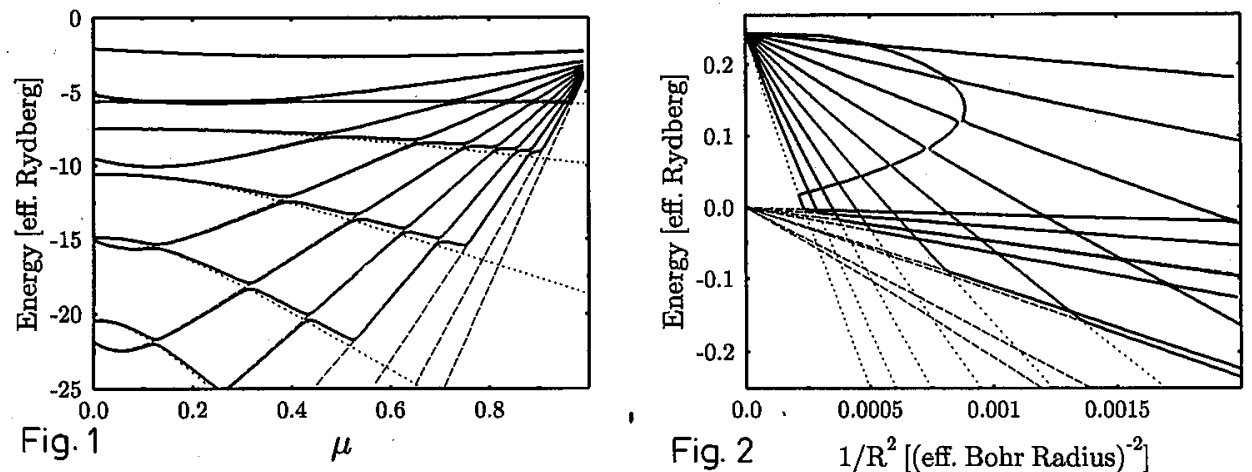

Fig. 1. Hole energy spectrum of the $J=1 / 2$ even states as a function of $\mu$. The dashed lines and the dotted ones correspond to the $\Gamma_{8}$ and SO states, calculated without the mixing effect. The spin-orbit splitting $\Delta=5 R^{*}$, the dot diameter $R=4 a^{*}$.

Fig. 2. Hole energy spectrum of the $J=3 / 2$ even states as a function of $(1 / R)^{2}$ for $\mathrm{CuCl}$. The dashed lines and the dotted ones correspond to the $\Gamma_{8}$ and SO states calculated without mixing. The highest value of the abscissa is equivalent to $R=60 \AA$.

be observed (Fig. 2), caused by the admixture of the wave functions with imaginary $k$ values to the $\mathrm{SO}$ ones.

The presented model can be easily extended to the more realistic case of a finite potential barrier.

\section{Acknowledgment}

We are indebted to Helena Tsitsishvili for stimulating discussion.

\section{References}

[1] L.E. Brus, J. Chem. Phys. 80, 4403 (1984).

[2] Y. Kayanuma, Phys. Rev. B 38, 9797 (1988).

[3] A.I. Ekimov, A.A. Onusenko, A.G. Pluhin, A.L. Efros, Zh. Eksp. Teor. Fiz. 88, 1490 (1985).

[4] J.B. Xia, Phys. Rev. B 40, 8500 (1989).

[5] K.J. Vahala, P.C. Sercel, Phys. Rev. Lett. 65, 239 (1990).

[6] P.C. Sercel, K.J. Vahala, Phys. Rev. B 42, 3690 (1990). 